\title{
Sex Hormone and Sex Hormone Receptors Modulation of Exercise-induced Neurogenesis
}

\author{
Peiyi Liu \\ Tianjin No.1 High School, Tianjin, 300051, China, Ninaliu0502@126.com
}

\begin{abstract}
Adult hippocampal neurogenesis (AHN) has been widely studied in many aspects. AHN is showed to have causal relationship with depression and anxiety. Experimental data showed that sex hormones and sex hormone receptors can be a factor that modulates AHN. However, few studies have focused on sex hormones' effect on exercise-induced neurogenesis, which the detailed mechanism is unknown to people. In this paper, we designed the experiments to find a casual relationship between sex hormones (receptors) and exercise-induced neurogenesis. We utilized castration (TX) and ovariectomy (OVX) to control the hormone level within the rats' body. Noteworthy, we also tested how can the numerical ratios of androgen to estrogen and androgen receptors to estrogen receptors affect exercise-induced neurogenesis by using conditional gene knockout and Cre recombinase. If the results turn out to be consistent with the hypothesis that the level of sex hormones and sex hormone receptors have influences on exercise-induced neurogenesis and depression-like behavior, we can put forward a treatment on anxiety disorders concerning the combination of exercise and sex hormone regulation.
\end{abstract}

Keywords: exercise-induced neurogenesis, sex hormones, sex hormone receptors, depression-like behavior.

\section{INTRODUCTION}

Adult hippocampal neurogenesis (AHN) is the capability of the adult mammalian brain to generate new neurons. Researches had proven that neurogenesis is associated with depression and anxiety. When the level of neurogenesis is enhanced, anxiety and depression-like behavior will be lowered. [1] Multiple factors can modulate AHN. Exercise, as one of the modulators, can increase the level of neurogenesis and is widely considered as a treatment for anxiety disorders. The detailed mechanism of this exercise-induced neurogenesis, however, has yet to be clear [2, 3]. As the database accumulated, ample studies uncover that sex fills in as a sectional mediator on the impacts of activityinduced benefits (e.g., lowered depressive symptoms, enhanced memory, enlarged hippocampal volume), which is indicated by the differentially affected neural and behavioral results $[4,5]$. Intense studies focused on the mechanism of sex hormones (specifically androgen and estrogen) regulating base-line adult-born neurogenesis. These studies discussed the sex hormones' significant role as neurogenic factors and multifaceted impact on different stages within the newborn neurons' maturation. [6-10] Androgen and estrogen are synthesized in both males and females, but in different volumes and play different roles by binding to their specific receptors (AR, ER $\alpha, E R \beta)$. [11] Some researches contend that these sex hormone receptors can modulate neurogenesis as well [12]. Moreover, assert that "The current study aimed at investigating the possibility that a combination of sex hormones and exercise has synergistic effects on neural plasticity in Transient Congenital Hypothyroidism rats [13]."

So, can sex hormones and their receptors affect exercise-induced neurogenesis? The purpose of this study is to evaluate the effects of Androgen and Estrogen and their receptors on exercise-induced neurogenesis. Our hypothesis is that: first, androgen, estrogen and their respective receptors' level have effects on exerciseinduced neurogenesis. Second, the numerical ratio of androgen/estrogen level and AR/ER impact exerciseinduced neurogenesis. Compared to other studies, our experiments not only test the impact of single sex hormone, but also considered the numerical ratio of the level of androgen to estrogen and the ratio of numbers of AR to ER as factors that may influence the exerciseinduced neurogenesis. If the result shows an effect of androgen and estrogen levels on neurogenesis enhanced by exercise, this study may hopefully be helpful with improving the anti-depressant effect performed by exercise, for we can modulate sex hormone level to reach a status under which exercise can maximize its effectiveness. 


\section{EXPERIMENTAL APPROACH}

\subsection{Sex influences exercise-induced neurogenesis. Exercise-induced neurogenesis is further enhanced in females.}

In order to test the hypothesis, we utilize SpragueDawley rats of equal number of males and females. Half of the males are treated with castration (TX), and half of the females are treated with ovariectomy (OVX). TX significantly reduces the circling androgen level in male mice $[14,15]$, while OVX lower the circling estrogen level along with the sum of estrogen receptors [16]. First, we divided the normal male and female rats into two groups, one with voluntary wheel running and one without. (Fig.1) (all figures are hypothesized data) We represent newborn neurons by the expression level of $\mathrm{Ki} 67+$ cells. If the result is consistent with the hypothesis, the female group will have a higher number of increased Ki67+markers than that increased in controls, suggesting that females result in a higher level of exercise-induced neurogenesis. Then we perform the tail suspension test (TST) to the four groups of rats to assess the number of depressive-like behaviors. (Fig.1b) The longer the total immobility of a rat, the more stressed it is [17]. If it is as expected, the total immobility of the female group will decrease more drastically after exercise than the male group. The data can also reapprove that exercise can increase neurogenesis and alleviate depressive-like behaviors. However, if the differences are the same, it means that neurogenesis only increased due to exercise instead of being related to sex difference. If the male group has a greater difference, it can be concluded that males result in a higher level of exercise-induced neurogenesis.

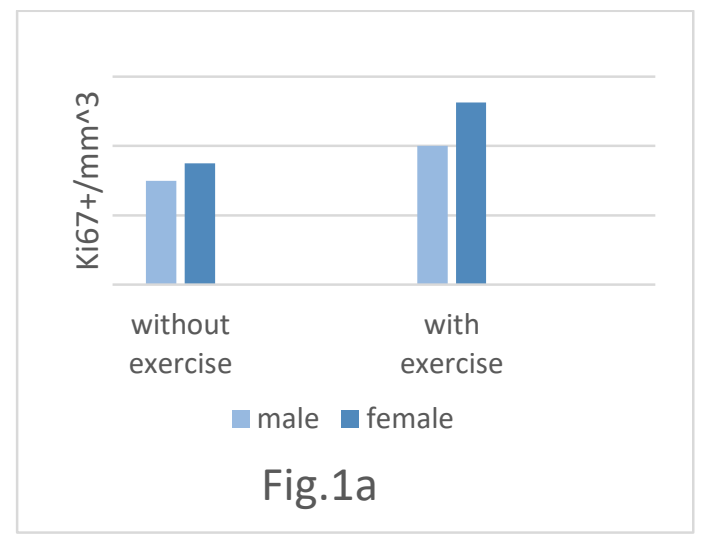

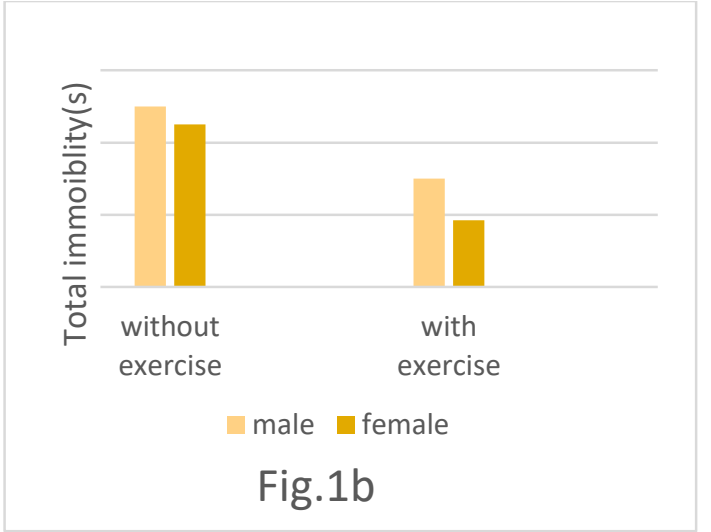

Fig.1 Exercise can further enhance neurogenesis level in female. a Quantification of Ki67+ dentate gyrus cells. b

Total immobility time recorded from tail suspension

test, a measurement for depression-like behavior.

\subsection{Sex hormones modulate exercise-induced neurogenesis. Estrogen enhances exercise- induced neurogenesis.}

If the data is consistent with the hypothesis in experiment one, so what causes the anti-depressant effect of exercise to react differently with sex? To answer this question, experiment two is designed to prove the hypothesis that sex hormones, specifically androgen and estrogen, cause this difference. To explore the effects of sex hormones on exercise-induced neurogenesis, we divided the mice into eight groups, four exercise groups and four non-exercise groups, which were then divided into normal male, normal female, TX male, and OVX female. The experimental data, shown in Fig.2, is consistent with the experimental conjecture. The presence of estrogen increases exercise-induced neurogenesis and alleviates anxiety, indicated by a further increase in the number of $\mathrm{Ki} 67+$ cells and a shorter immobility time period in the TST. The estrogen-free OVX group has fewer increases in Ki67 markers than the other three groups with a certain estrogen level. Specifically, the normal female group with the highest estrogen levels among these three groups should have the highest increases in Ki67 markers and the most significant decrease in the total immobility period, suggesting that estrogen promotes the effect of exerciseinduced neurogenesis more than androgen. The results could also be used to determine whether androgen has a positive or negative effect on the neurogenesis. If the results do not match the hypothesis that the increase in neurogenesis in normal female or normal male groups is not different from that in other groups, then exerciseinduced neurogenesis is possibly not related to estrogen and androgen level. As expected, the TST figure will follow the same trend as neurogenesis. The Normal female group had fewer seconds of immobility than the other three groups, suggesting that estrogen has a better effect on reversing depressive-like behavior with exercise. The OVX group reduces the minimum immobility time. 


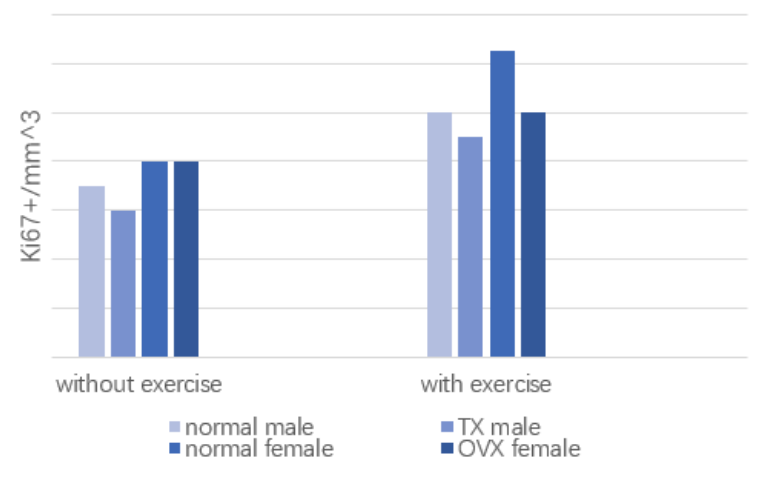

Fig.2a

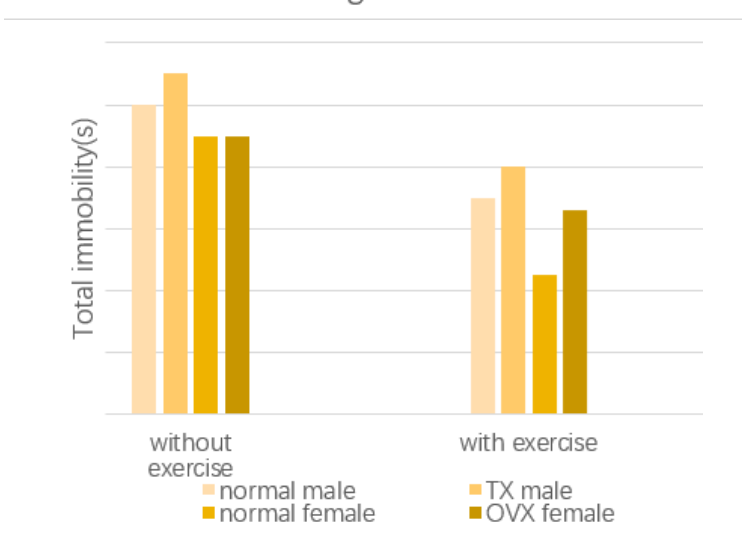

Fig.2b

Fig.2 Estrogen further enhances exercise-induced neurogenesis than androgen. a Quantification of Ki67+ dentate gyrus cells. b Total immobility time recorded from tail suspension test, a measurement for depressionlike behavior.

\subsection{The numerical ratio of androgen/estrogen can modulate exercise-induced neurogenesis. The ratio is negatively related to neurogenesis levels.}

Suppose the previous results confirm that estrogen improves exercise's promotive effect on neurogenesis, while androgen inhibits or stabilizes the effect. Could it be an evidence for claiming that the numerical ratio of estrogen/androgen also has an effect on exercise-induced neurogenesis? We re-injected different doses of estrogen and androgen into the TX and OVX mice to achieve the goal of regulating the estrogen/androgen ratio (figure $3 \mathrm{a}$ ). The injections are $100 \%$ androgen; $75 \%$ androgen and $25 \%$ estrogen; $25 \%$ androgen and $75 \%$ estrogen; $100 \%$ estrogen respectively. If the results of the experiment are in line with the conjecture, Ki67 marker treated by dose four should be increased more than dose three, and dose three should be more than dose two, dose two more than dose one (Fig3b), because estrogen increases exerciseinduced neurogenesis, and estrogen level increases in turn from dose 1 to dose 4 . Taking all these together, the estrogen/androgen ratio is suggested to have a negative regulation on exercise-induced neurogenesis.

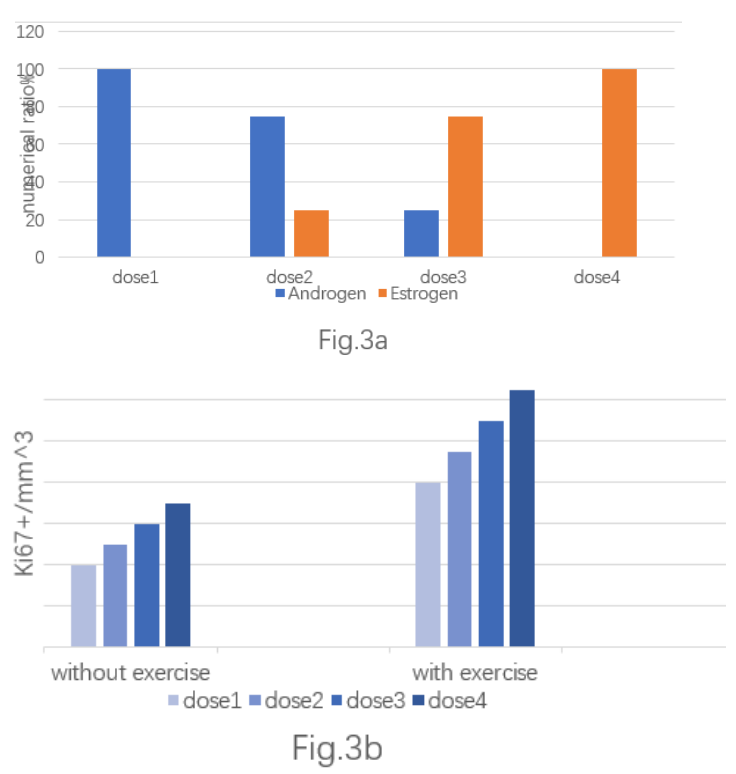

Fig.3 Lower androgen/estrogen ratio results in higher level of exercise-induced neurogenesis. a Numerical ratio representing different levels of androgen and estrogen in 4 different doses of injection. b Quantification of Ki67+ dentate gyrus cells under different doses.

\subsection{ER upregulates exercise-induced neurogenesis. The numerical ratio of $A R / E R$ has a negative correlation with exercise- induced neurogenesis.}

Based on experimental evidence that sex hormones upregulate the expression of Ars and ERs [18, 19], we hypothesize that the number of receptor proteins alone is sufficient to affect exercise-induced neurogenesis. We use AR antagonist and ER antagonist to investigate this effect. Firstly, we use AR antagonist to silence AR expression and measure the number of Ki67 + cells. Then the ER antagonist is used to silence the ER expression, and the number of KI67 + cells is assessed. The numbers are compared with the control group. If the ARsuppressed group causes a larger increase in Ki67 markers after exercise, it is suggested that AR inhibits exercise-induced neurogenesis. Similarly, if the ERsuppressed group further increases the Ki67 markers after exercise, we can conclude that ER inhibits exerciseinduced neurogenesis. No difference between the two groups indicates that the number of receptor proteins does not affect the level of exercise-induced neurogenesis. Suppose that the number of receptor proteins has an effect on exercise-induced neurogenesis. After silencing the receptors, we take serum hormone assays to test estrogen and androgen level. If the hormone level is not altered, we can demonstrate that the number of Ars and ERs itself is sufficient to regulate exercise-induced neurogenesis; estrogen and androgen do not take responsibility for how ARs and ERs affect exercise-induced neurogenesis. But if hormone level changes, it is likely that estrogen and androgen cause the change, rather than the number of 
receptor proteins. In that case, we should find a way to maintain the normal hormone level before measuring the effects of AR and ER on exercise-induced neurogenesis.

Equivalent to previous experiments, we also hypothesize that the $\mathrm{AR} / \mathrm{ER}$ ratio has an effect on exercise-induced neurogenesis. There are two types of $\mathrm{ER}, \mathrm{ER} \alpha$ and ER $\beta$. They are products of independent genes. ER $\alpha$ is located on chromosome $14 q 22-24$ and $\mathrm{ER} \beta$ is located on chromosome $6 \mathrm{q} 25.1$ [20, 21]. To modulate the $\mathrm{AR} / \mathrm{ER}$ ratio, we use inducible Cre recombinase to conduct conditional gene knockout of one set of ER genes. (Fig.4b) Suppose the initial ratio index of $\mathrm{AR} / \mathrm{ER}$ is $\mathrm{X}$, then after the recombination, the index turns to $2 \mathrm{X}$, for we have silenced half of the ER. (Fig.4a) After assessing the recombination efficacy of the transgenes, we use immunofluorescent staining to examine the exact number of the normal index ratio and the ratio after recombination [22]. Subsequently, we can analyze how different numerical ratios of AR/ER regulate exercise-induced neurogenesis. The expected result is that the control increases more than the mutated group, for the control has more ERs. As described in the previous experiment, we still need to conduct serum hormone assays to test whether the hormone level ratio itself has an effect on exercise-induced neurogenesis.

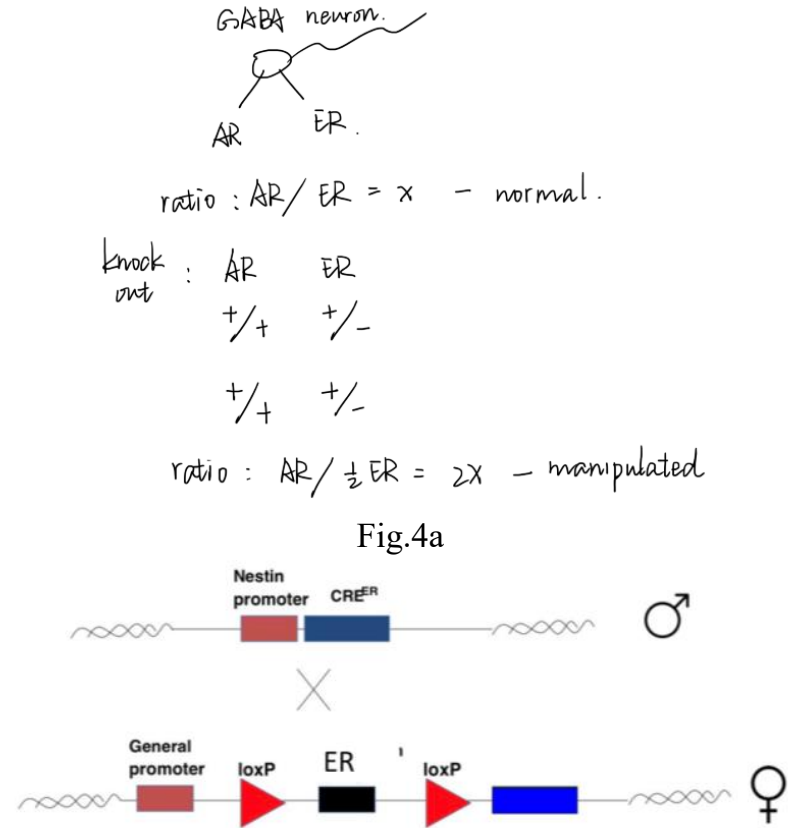

Fig. $4 b$

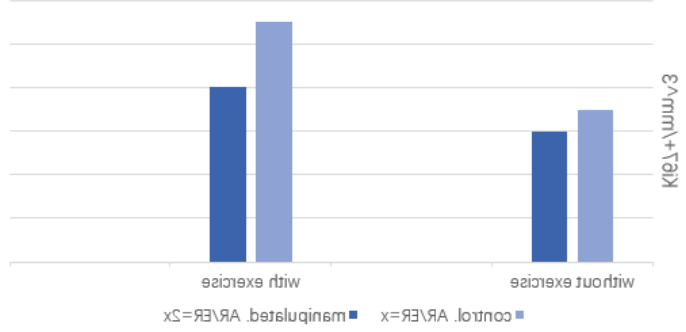

Fig. $4 \mathrm{c}$

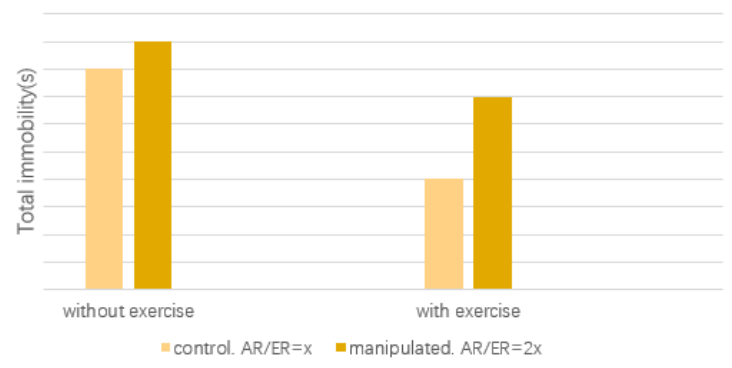

Fig. $4 d$

Fig.4 Lower AR/ER ratio results in a higher level of exercise-induced neurogenesis. $a$ and $b$ Representative images showing how the AR/ER ratio is manipulated. c

Quantification of Ki67+ dentate gyrus cells. d Total immobility time recorded from tail suspension test, a measurement for depression-like behavior.

To verify how long-lasting those effects on exerciseinduced neurogenesis are, each group of rats is anaesthetized and their hippocampus are removed to be analyzed of AHN after 1 hour, 1 day, 7 days, and 30 days respectively. It is reasonable to hypothesize that there will be a decreasing trend of the data, but we cannot actually suggest the detailed information about the data, for example, will the level of neurogenesis decrease for some time and remains at a constant level, or will it decrease to its original level within 30 days? We do not have solid evidence to infer these details. Nevertheless, obtaining and analyzing these data will provide us with information about how effective this combination of exercise and hormone treatment will be and the appropriate timing to regulate the treatment.

\section{CONCLUSION}

In this study, we first expected to observe that the number of Ki67+ cells increases more after exercise in the normal female group, which indicates that sex can affect the level of exercise-induced neurogenesis. Then, we modulate the hormone level of the rats and hypothesize that the androgen and estrogen level, single and their ratio cause this difference. After that we considered another factor: AR, ER, and their numerical ratio. If the results are consistent with the hypothesis, the data should display a positive correlation between the number of ER and Ki67+ cells. Lastly, we test how longlasting are these treatment's effects on exercise-induced neurogenesis. Few studies have been conducted to test the possibilities of factors that influence exercise-induced neurogenesis. If the experimental data demonstrate that the combination of exercise and sex hormone and sex hormone receptors has an interdependent effect on neurogenesis and depressive-like behaviors, we may find high qualitied, more effective treatments for anxiety disorders.

However, some questions need further research. Age, species, experience, exercise intensity and exposure frequency to sex hormones are proved to be a factor that determines adult-born neurogenesis. Also, plenty of hormones can lead to changes in various stages of the 
neural differentiation cascade $[6,7,12,23,24]$. They may have an effect on exercise-induced neurogenesis as well. Moreover, this study does not uncover the direct relationship between sex hormone level and the number of sex hormone receptors located in the hippocampus. We only test how can these two factors affect exerciseinduced neurogenesis levels respectively. However, the mechanism of how these factors cause the effect is still not fully understood. Further studies targeting this issue may lead to more effective antidepressant treatments.

\section{REFERENCES}

[1] Hill, A. S., Sahay, A., \& Hen, R. (2015). Increasing adult hippocampal neurogenesis is sufficient to reduce anxiety and depression-like behaviors. Neuropsychopharmacology, 40(10), 2368-2378. https://doi.org/10.1038/npp.2015.85

[2] Yau, S.-yu, Gil-Mohapel, J., Christie, B. R., \& So, K.-fai. (2014). Physical exercise-induced adult neurogenesis: A good strategy to prevent cognitive decline in neurodegenerative diseases? BioMed Research International, 2014, 1-20. https://doi.org/10.1155/2014/403120

[3] Hueston, C. M., Cryan, J. F., \& Nolan, Y. M. (2017). Stress and adolescent hippocampal neurogenesis: Diet and exercise as cognitive modulators. Translational Psychiatry, 7(4). https://doi.org/10.1038/tp.2017.48

[4] Gorham, L. S., Jernigan, T., Hudziak, J., \& Barch, D. M. (2019). Involvement in Sports, hippocampal volume, and depressive symptoms in children. Biological Psychiatry: Cognitive Neuroscience and Neuroimaging, 4(5), 484-492. https://doi.org/10.1016/j.bpsc.2019.01.011

[5] Loprinzi, P. D., Day, S., Hendry, R., Hoffman, S., Love, A., Marable, S., McKee, E., Stec, S., Watson, H., \& Gilliland, B. (2021). The effects of acute exercise on short- and long-term memory: Considerations for the timing of exercise and phases of memory. Europe's Journal of Psychology, 17(1), 85-103. https://doi.org/10.5964/ejop. 2955

[6] Jorgensen, C., \& Wang, Z. (2020). Hormonal regulation of Mammalian Adult Neurogenesis: A Multifaceted Mechanism. Biomolecules, 10(8), 1151. https://doi.org/10.3390/biom10081151

[7] Galea, L. A. M. (2008). Gonadal hormone modulation of neurogenesis in the dentate gyrus of adult male and female rodents. Brain Research Reviews, 57(2), 332-341. https://doi.org/10. 1016/j. brainre srev. 2007. 05. 008

[8] Galea, L. A., Wainwright, S. R., Roes, M. M., Duarte-Guterman, P., Chow, C., \& Hamson, D. K. (2013). Sex, hormones and neurogenesis in the hippocampus: Hormonal modulation of neurogenesis and potential functional implications. Journal of Neuroendocrinology,
25(11),

1039-1061.

https://doi.org/10.1111/jne. 12070

[9] Spritzer, M. D., \& Roy, E. A. (2020). Testosterone and adult neurogenesis. Biomolecules, 10(2), 225. https://doi.org/10.3390/biom10020225

[10] Yagi, S., \& Galea, L. A. (2018). Sex differences in hippocampal cognition and neurogenesis. Neuropsychopharmacology, 44(1), 200-213. https://doi.org/10.1038/s41386-018-0208-4

[11] Hammes, S. R., \& Levin, E. R. (2019). Impact of estrogens in males and androgens in females. Journal of Clinical Investigation, 129(5), 1818 1826. https://doi.org/10.1172/jci125755

[12] Selakovic, D., Joksimovic, J., Jovicic, N., Mitrovic, S., Mihailovic, V., Katanic, J., Milovanovic, D., Pantovic, S., Mijailovic, N., \& Rosic, G. (2019). The impact of Hippocampal sex Hormones receptors in modulation of Depressive-Like behavior Following chronic ANABOLIC Androgenic steroids and Exercise protocols in rats. Frontiers in Behavioral Neuroscience, 13. https://doi.org/10.3389/fnbeh.2019.00019

[13] Derafshpour, L., Saboory, E., Vafaei, A. A., Rashidy-Pour, A., Roshan-Milani, S., Rasmi, Y., Panahi, Y., \& Sameni, H. (2018). Interactive effects of exercise, sex hormones, and transient congenital hypothyroidism on long-term potentiation in hippocampal slices of rat offspring. Basic and Clinical Neuroscience Journal. https://doi.org/10.32598/bcn.9.10.170

[14] Siegel, H. I. (1985). Male sexual behavior. The Hamster, 191-206. https://doi.org/10.1007/978-14757-0815-8_9

[15] Wood, R. I., \& Newman, S. W. (1995). Hormonal influence on neurons of the mating behavior pathway in male hamsters. Neurobiological Effects of Sex Steroid Hormones, 3-39. https://doi.org/10.1017/cbo9780511529832.003

[16] Rose'Meyer, R. B., Mellick, A. S., Garnham, B. G., Harrison, G. J., Massa, H. M., \& Griffiths, L. R. (2003). The measurement of adenosine and estrogen receptor expression in rat brains following ovariectomy using quantitative $\mathrm{pcr}$ analysis. Brain Research Protocols, 11(1), 9-18. https://doi.org/10.1016/s1385-299x(02)00219-2

[17] Can, A., Dao, D. T., Terrillion, C. E., Piantadosi, S. C., Bhat, S., \& Gould, T. D. (2011). The tail suspension test. Journal of Visualized Experiments, (58). https://doi.org/10.3791/3769

[18] Ing, N. H., \& Belen Tornesi, M. (1997). Estradiol up-regulates estrogen receptor and progesterone receptor gene expression in specific ovine uterine cells1. Biology of Reproduction, 56(5), 12051215.

https://doi.org/10.1095/biolreprod56.5.1205

[19] Kostic, T. S., Stojkov, N. J., Bjelic, M. M., Mihajlovic, A. I., Janjic, M. M., \& Andric, S. A. (2011). Pharmacological doses of Testosterone Upregulated androgen receptor and 3-BETAHYDROXYSTEROID Dehydrogenase/delta-5- 
delta-4 Isomerase and impaired Leydig Cells Steroidogenesis in Adult Rats. Toxicological Sciences, 121(2), 397-407. https://doi.org/10.1093/toxsci/kfr063

[20] Enmark, E., Pelto-Huikko, M., Grandien, K., Lagercrantz, S., Lagercrantz, J., Fried, G., Nordenskjöld Magnus, \& Gustafsson Jan-Åke. (1997). Human estrogen receptor $\beta$-gene structure, chromosomal localization, and expression pattern1. The Journal of Clinical Endocrinology \& Metabolism, 82(12), 42584265. https://doi.org/10.1210/jcem.82.12.4470

[21] Menasce, L. P., White, G. R. M., Harrison, C. J., \& Boyle, J. M. (1993). Localization of the estrogen RECEPTOR Locus (ESR) to CHROMOSOME 6q25.1 by fish and a simple Post-fish banding technique. Genomics, 17(1), 263-265. https://doi.org/10.1006/geno.1993.1320

[22] Zaqout, S., Becker, L.-L., \& Kaindl, A. M. (2020). Immunofluorescence staining of Paraffin sections step by step. Frontiers in Neuroanatomy, 14. https://doi.org/10.3389/fnana.2020.582218

[23] Denny, C. A., Kheirbek, M. A., Alba, E. L., Tanaka, K. F., Brachman, R. A., Laughman, K. B., Tomm, N. K., Turi, G. F., Losonczy, A., \& Hen, R. (2014). Hippocampal memory traces are Differentially modulated by EXPERIENCE, time, and adult neurogenesis. Neuron, 83(1), 189-201. https://doi.org/10.1016/j.neuron.2014.05.018

[24] Kuhn, H. G., Toda, T., \& Gage, F. H. (2018). Adult hippocampal neurogenesis: A coming-of-age story. The Journal of Neuroscience, 38(49), 10401-10410.

https://doi.org/10.1523/jneurosci.2144-18.2018 